\title{
OUTDOOR WORKERS AND SUN PROTECTION: KNOWLEDGE AND BEHAVIOUR
}

\author{
Dr J ane Cioffi, Professor Lesley Wilkes and J ess Hartcher-O'Brien \\ The University of Western Sydney
}

\section{INTRODUCTION}

Due to Australia's close proximity to the equator New South Wales (NSW) has very high levels of solar ultra violet radiation (UVR) (Gies et al., 1997). A major effect of UVR is skin cancer. In Australia, new cases of skin cancer outnumber all other forms of cancer by more than three to one. Currently there are around 1,300 deaths each year from this disease, with 2,807 new cases of skin melanoma in NSW (The Cancer Council NSW and NSW Health Department, 2001). For adult males (20-54 years) melanoma of the skin is the most common form of cancer (Coates and Armstrong, 2000; Marks et al., 1993).

The single most important modifiable risk factor for skin cancer is unprotected exposure to solar ultraviolet radiation. A group at high risk of skin cancer is adults who have high cumulative exposure to UVR in the workplace. Outdoor workers are part of this high risk group and are targeted in the Skin Cancer Prevention Strategic Plan for NSW 2001-2005 (The Cancer Council NSW and NSW Health Department, 2001). This study describes outdoor construction workers' knowledge and sun protective behaviour associated with their risk of skin cancer.

\section{LITERATURE REVEW}

Some studies were found that addressed outdoor workers in Australia (Dobbinson and Knight, 2001; Parisi et al., 1999; Kimlin et al., 1998; Airey et al., 1997; Green et al., 1996; Wong et al., 1996; Girgis et al., 1994; Borland et al., 1991). These studies were carried out mostly in Queensland, with two in Victoria and another in NSW. Outdoor workers who participated included farmers, Telecom lines staff, and tradespersons. The focus of five of these studies was exposure to UVR (Parisi et al., 1999; Kimlin et al., 1998; Airey et al., 1997; Green et al. 1996; Wong et al., 1996) with the remaining studies focusing on the effects of an interventions on outdoor workers' sun protective behaviour (Girgis et al., 1994; Borland et al., 1991) and organisational change (Dobbinson and Knight, 2001). No study was found that had surveyed outdoor workers in NSW to assess their knowledge and sun protective behaviour associated with their risk of skin cancer.

In Australia, sun protection precautionary measures were self-reported by a majority of people $(82 \%$ of males and $83 \%$ of females) (Australian Bureau of Statistics, 1999). Specifically in NSW, $59 \%$ of males protected themselves against the sun with a cap or hat, 38.6\% applied sunscreen and $54.2 \%$ reported having a skin check NSW Health Department, 2000). Other Australian studies have shown males as a subgroup tend to spend more time in the sun and employ fewer sun-protective measures except for wearing a hat (Hill et al., 1992; Scholfield et al., 2001; Wentworth Area Health Service, 2000). Hence, males in the community do not generally practise sun protection at acceptable levels.

Unprotected sun-exposure has been found to be treated as advantageous where the prevalent perception correlates a tan with health, 'looking sexy' and 'mood' improvement (Sahn, 1995). In such a context the inherent dangers that come with sun exposure are not al ways acknowledged. This attitude, if present in outdoor workers, would influence behaviour and further increase their risk of skin cancer as resistance to sun protection strategies has been found to be associated with a 'sun' culture (Dobbinson and Knight, 2001).

In the occupational context of outdoor workers, the implications of the high levels of sun exposure are an occupational health and safety concern that has legislative support in NSW. Recommendations for workplace sun protection outlined in the Skin Cancer Prevention Strategic Plan for NSW 2001-2005 are engineering controls including provision and use of shade; administrative controls including rescheduling work to less UV intense times; and personal protection including use of protective clothing and equipment such as long sleeved protective shirts and trousers, broad brimmed hats, 
sunglasses and sunscreen (The Cancer Council NSW and NSW Health Department, 2001). Organisations, therefore, have a responsibility to support workers' sun protection. Over the last five years local government and the construction industry in NSW have been targeted with the development of policies and implementation of programs for sun protection (The Cancer Council NSW and NSW Health Department, 2001). No evaluation of these programs was found. However, an evaluation of a program in Victoria aimed at achieving positive policy and behavioural changes in workplace settings found $40 \%$ had a sun protection policy in place, $18 \%$ were developing a policy. Personal protective equipment was the most common strategy and changes to work schedules or training were less commonly provided. Problems were being experienced with compliance and policy implementation including resistance to the provision and use of protective clothing (especially in the heat), a sun culture and lack of awareness or denial of the risks of UV exposure (Dobbinson and Knight, 2001). This suggests changing workplace culture may meet resistance and may require careful strategic planning.

However, in Australia two studies have shown the sun protection behaviour of outdoor workers can be significantly increased (Girgis et al., 1994; Borland et al., 1991). The interventions used in the randomised control trial (Girgis et al., 1994) was an individual skin screening by a dermatologist and an education session, and in the quasi experimental study, a campaign using marketing techniques (Borland et al., 1991). This suggests that health education programs of similar design would be effective in achieving changes in the sun protective behaviour of outdoor workers.

Information about skin cancer and sun protection provides people with the opportunity to understand the value of sun protection. According to the Skin Cancer Prevention Strategic Plan for NSW 2001-2005, mass media, particularly television advertising, has played an important role in the last 20 years in raising and maintaining high levels of community awareness of the importance of sun protection (The Cancer Council NSW and NSW Health Department, 2001). WorkCover in NSW has been encouraging workplaces to adopt structures and practices to improve the safety and health of employees. A resultant acceptance and use of protective clothing has been attributed to this intervention (The Cancer Council NSW and NSW Health Department, 2001). No study was found that reported outdoor workers' level of awareness of the dangers of skin cancer as measured by knowledge and sun protective work behaviour.

\section{METHOD}

Design. A survey design using questionnaires was used to determine outdoor workers level of awareness of the dangers of skin cancer as measured by knowledge of and attitudes to skin cancer and sun protection behaviour.

Sample: Outdoor workers ( $\mathrm{n}=142$ ) working on construction sites in northwest and western Sydney who consented to complete the questionnaire formed the convenience sample. Only a few workers who were approached declined to join the study, usually giving tight schedules as the reason.

Instrument: A short close-ended questionnaire was designed to address behaviour (nine items), shade equipment (one item), sources of information (two items) and knowledge (six items) to determine, respectively, use of protective measures and shade equipment available, sources of information and basic knowledge of skin cancer and sun protection. Some items were adapted from the 1997 NSW Health Survey Questionnaire (NSW Health Department, 2000) and others were developed based on study findings (Moehrle et al., 2000; Rosenthal et al., 1988). The questionnaire was deliberately designed to take no more than five minutes to administer to each worker on site.

Data Collection Procedure. At each site workers who volunteered to join the study most usually requested that each item on the questionnaire be read to them whilst they continued to work. They then indicated their responses, which were recorded by the research assistant.

Data Analysis. Frequencies and percentages were used to summarise responses to questionnaire items.

\section{RESULTS}

Of the 142 outdoor construction workers who participated in the study, 139 were males and three were females. Respondents were from a variety of trades including bricklaying, plumbing and painting and most were in the 31 to 40 years age range. 
Table 1: Frequencies and percentages for sun protective behaviour at work

\begin{tabular}{|l|l|l|l|l|}
\hline Item & $\begin{array}{l}\text { Never } \\
f=(\%)\end{array}$ & $\begin{array}{l}\text { Occasional ly } \\
f=(\%)\end{array}$ & $\begin{array}{l}\text { Frequently } \\
f=(\%)\end{array}$ & $\begin{array}{l}\text { Always } \\
f=(\%)\end{array}$ \\
\hline $\begin{array}{l}\text { Wore a } \\
\text { brimmed hat }\end{array}$ & $50(35)$ & $15(11)$ & $17(12)$ & $60(42)$ \\
\hline $\begin{array}{l}\text { Wore a } \\
\text { baseball cap }\end{array}$ & $59(41)$ & $27(19)$ & $18(13)$ & $38(27)$ \\
\hline $\begin{array}{l}\text { Wore } \\
\text { sunglasses }\end{array}$ & $27(19)$ & $28(20)$ & $30(21)$ & $57(40)$ \\
\hline $\begin{array}{l}\text { Wore a sleeved } \\
\text { shirt }\end{array}$ & $107(75)$ & $19(13)$ & $4(3)$ & $11(8)$ \\
\hline $\begin{array}{l}\text { Used sun } \\
\text { lotion }\end{array}$ & $35(25)$ & $57(40)$ & $19(13)$ & $30(21)$ \\
\hline $\begin{array}{l}\text { Used a shade } \\
\text { device\# }\end{array}$ & $122(86)$ & $12(9)$ & $6(4)$ & $1(1)$ \\
\hline
\end{tabular}

\# 1 non response present

Twenty six percent reported getting sunburnt frequently/always, $48 \%$ occasionally and $25 \%$ never.

Sun protective work behaviours reported by workers showed they frequently/always wore a brimmed hat $(56 \%)$, a baseball cap $(50 \%)$, sunglasses $(61 \%)$, sleeved shirt $(11 \%)$, sun lotion (34\%) and used a shade device $(5 \%)$ to protect themselves against UVR (See Table 1). Workers showed they did not exclusively use a brimmed hat or a cap at work but were inclined to use them interchangeably. Other protective behaviour reported was the use of machinery fitted with a shade device $(14 \%)$, awareness of the UV index in the daily weather report (65\%) and a skin check in the last 12 months (33\%). Nineteen percent of the workers had previously had a skin lesion removed.

Workers showed they knew working without sunglasses caused eye damage (85\%), their risk of skin cancer was increased by working in middle of the day (94\%), and important areas to cover are head and chest (82\%). However, fewer workers knew sweating increased their risk of sunburn (43\%). Forty four percent of workers considered a tanned body was healthy and $72 \%$ stated a tan improved appearance. Sources from which workers had received information about skin cancer were television $(78 \%)$, radio $(0 \%)$, newspaper (35\%) with others being pamphlets (6\%), local doctor (4\%), parents (4\%) and WorkCover (3\%). However, only $44 \%$ reported receiving information about skin cancer recently, that is during the last year.

\section{Discussion}

The main findings showed some form of head apparel and sunglasses were the most common sun protection used by workers. However, their use was not as high as would be desirable. About a third applied sun lotion and there was only poor use of sleeved shirts and shade devices. The low use of sun Iotion may be attributed to workers' perception that it could be harmful. A comment from one worker reflects this view, "Sunscreen is more unhealthy for you than skin damage ... accumulates in liver. So I won't wear it." Application of sunscreen by males was similar to that reported in NSW Health Survey (1997) with the use of a cap or hat being slightly below (NSW Health Department, 2000). This suggests male sun protection behaviour has altered little in the last five years. Personal strategies employed by outdoor workers need to be increased to reduce high cumulative exposure in the workplace.

Workers' knowledge of sun protection and risk was on the whole quite high which may reflect information they had received about skin cancer mostly from television, radio and the newspaper, respectively. The recency of receiving this information was less than desirable as just below half had received this information in the last year. Knowledge, however, did not directly translate into sun protective behaviour. In addition, nearly three-quarters of the workers reported some episodes of sunburn and a marked number of workers perceived a tan to be healthy and attractive. Workers commented for example that “...it's healthy 
"cause when you look good, you feel good." and "A tanned body is healthy. We're in Australia!" This suggests there is some resistance to the acknowledgement of the importance of the information received. Worker resistance similar to this was previously found by Dobbinson and Knight (2001) and Borland et al. (1991). Such resistance may be due to factors such as a lack of motivation and a competing value or norm such as the 'bronze image' culture.

The receipt of skin checks by a third of the workers in the last year indicates some engagement in screening activities. Skin checks reported by workers were much lower than that reported by males in NSW in 1997 (NSW Health Department, 2000). A higher uptake of skin screening in these workers needs to be encouraged through health education programs in the workplace that are aimed at workers accepting responsibility for their own risk assessments.

Accessing workers directly on site was found to be most successful. Prior to this an initial distribution approach was attempted through the head offices of main construction firms operating in the area, however, this was not successful, largely due to the subcontracting of work by these firms. The use of a short questionnaire was found to elicit strong participation from workers. Additional items to consider in future questionnaires could include the estimated period of time employed as outdoor worker, daily average working hours and details of time of day exposed to sun. This would provide insight into workers' cumulative exposure risk. Items from other questionnaires and studies addressing sun protection were used; however, the validity and reliability of the questionnaire were not tested. The generalisability of the findings to all outdoor workers is restricted as a convenience sample and only one type of outdoor worker was included.

Despite the limitations of this survey it can be considered that outdoor construction workers need to improve patterns of sun protective behaviour in the workplace. It is recommended that research needs to be planned to address issues that outdoor workers have with using sun protective strategies. Such studies need to provide further insight and understanding of outdoor workers and sun protection to inform future health promotion programs. Such programs maybe more effective if they are implemented on actual construction sites and include strategies that actively coach workers to use better sun protective practices.

Ethics approvals for this study were obtained from The University of Western Sydney Human Research Ethics Committee.

\section{ACKNOMLEDGMENTS}

To the construction workers who took part in the study.

To Ms Shantala Mohan for her support of the project.

\section{REFERENCES}

Airey, D.K., Wong, J.C., Fleming, R.A. and Meldrum, L. R. (1997) An estimate of the total UV-B exposure for outdoor workers during a south-east Queensland summer. Health Physics, 72, 544-9.

Australian Bureau of Statistics (1999) National Health Survey: Cancer related information. Special article. September.

Borland, R. M., Hocking, B., Godkin, G. A., Gibbs, A. F. and Hill, D. J . (1991) The impact of a skin cancer control education package for outdoor workers. Medical Journal of Australia, 154, 686-8.

Coates, M. S. and Armstrong, B. K. (2000) Cancer in NSW. Incidence and mortality 1997. NSW Cancer Council, Sydney.

Dobbinson, S, and Knight, K. (2001) Protecting workers from ultraviolet radiation in sunlight. Journal of Occupational Health Safety_Australia and New Zealand, 17, 587589.

Gies, P. H., Roy, C. R., Toomey, S. and McLennan, A. (1997) Protection against solar UV radiation. Sun Protection Seminar Papers. Australian Cancer Society.

Girgis, A., Sanson-Fisher, R. W., and Watson, A. (1994) A workplace intervention for increasing outdoor workers' use of solar protection. American Journal of Public Health, 84, 77-81.

Green, A., Battistutta, D., Hart, V., Leslie, D. and Weedon, D. (1996) Skin cancer in a subtropical Australian population: Incidence and lack of association with occupation. The Nambour Study Group. American J ournal of Epidemiology, 144, 1034-40. 
Hill, D., White, V., Marks, R., Theobald, T., Borland, R. and Roy, C. (1992) Melanoma prevention: Behavioural and nonbehavioural factors in sunburn among an Australian urban population. Preventive Medicine, 21, 654-669.

Kimlin, M. G., Parisi, A.V. and Wong, J. C. (1998) Quantification of personal solar UV exposure of outdoor workers, indoor workers and adolescents at two locations in south-east Queensland. Photodermatology, Photoimmunology, Photomedicine, 14, 7-11.

Marks, R., Staples, M., and Giles, G.G.(1993) Trends in non-melanocytic skin cancer treated in Australia: the second national survey. International J ournal of Cancer, 53, 585-590.

Moehrle, M., Koehle, W., Dietz, K. and Lischka, G. (2000). Reduction of erythema dose by sweating. Photodermatology, Photoimmunology, Photomedicine, 16, 260-262.

NSW Health Department (2000) Public Health Division, Report on the 1997 and 1998 NSW Health Surveys. http://www.health.nsw/gov.au/publicnswhs/sun_intro.htm.

Parisi, A. V., Meldrum, L.R., Wong, J .C., Aitken, J. and Fleming R. A. (1999) Lifetime ultraviolet exposure estimates for selected population groups in south-east Queensland. Physics in Medicine and Biology, 44, 2947-53.

Rosenthal, F.S., Phoon, C., Bakaaalian, A. E. and Taylor, H. R. (1988) The ocular dose of ultraviolet radiation to outdoor workers. Investigative Ophthalmology and Visual Science, 29, 649-56.

Sahn, E. (1995) Why teenagers embrace sun's dangers. In Skin cancer-The need for continuing education. Hill, M. Dermatology Nursing, 7, 220-222.

Schofield, P. E., Freeman, J . L., Dixon, H.G., Borland, R. and Hill, D.J . (2001) Trends in sun protection behaviour among Australian young adults, Australian and New Zealand J ournal of Public Health, 25, 62-65.

The Cancer Council NSW and NSW Health Department (2001) Skin Cancer Prevention Strategic Plan for NSW 2001-2005.

Wentworth Area Health Service (2000) Health profile: Wentworth Area Health Service 1993-1998 Penrith, NSW.
Wong, J.C., Airey, D.K., and Fleming, R.A. (1996) Annual reduction of solar UV exposure to the facial area of outdoor workers in southeast Queensland by wearing a hat. Photodermatology, Photoimmunology, Photomedicine, 12, 131-5. 